\title{
Is mandibular molar distalization feasible?
}

\author{
T. Dang ${ }^{1,3,5,6}$, J.-P. Forestier ${ }^{1,2,4,5,6}$, B. Thebault ${ }^{1,2}$ \\ 1 Docteur en chirurgie dentaire \\ 2 Spécialiste qualifié en ODF \\ 3 CECSMO 4 \\ $4 \mathrm{MCU}-\mathrm{PH}$ \\ 5 Université Paris 7 Denis Diderot \\ 6 Hôpital Pitié Salpêtriére AP-HP, Paris
}

\begin{abstract}
Introduction: The mandibular molar is considered the most difficult tooth to move. In certain clinical situations, it would seem useful to move it backward. Is that feasible? When would it be indicated? Is it successful, and if so how? Material and methods: We first review some fundamental principles and present an update of the literature on mandibular molar distalization, then analyze a retrospective series of 11 patients for whom mandibular molar distalization was planned. The movement achieved was studied on dental cephalometric superimposition with Delaire analysis. Results: the desired pure distal translation was achieved in 2 of the 11 cases; distalization was accompanied by coronary tip-back in 3 cases; in 4 cases, only coronary tipback was achieved, and apical tip-forward in 2 cases. Conclusion: Further studies are needed to determine optimally effective and reproducible distalization modalities for the mandibular molar.
\end{abstract}

\section{KEY WORDS}

Distalization, mandibular molar, bone screw, bone plate

\section{INTRODUCTION}

\section{Context}

Orthodontic displacement of the mandibular molar, other than extrusion, is reputed to be extremely difficult, due to the large root area and root anatomy ${ }^{3}$.

In certain clinical situations, however, extreme measures may be taken to avoid irreversible or risk-laden procedures such as extraction or orthognathic surgery.
Organizing the space within the dental arcade involves two essential factors: tooth size and arcade perimeter. The latter is determined by the anterior, lateral and posterior edges, and the space occupied by the arcade depends on 3D compensatory curves.

In adjusting tooth crowding, any extraction is usually performed in the sector where crowding is present, to limit and 
facilitate orthodontic movement in the freed space. For example, in severe anterior crowding associated to correction of mandibular incisor alveolar protrusion, the 1st premolars may classically be extracted. However, it might be interesting if the space freed by the absence of the 3rd molars could be transferred forward, and if the mandibular molars could be distalized.

While possibly indicated, class III surgical correction has a number of drawbacks or relative contraindications leading to abstention despite skeletal malocclusion. Over and above the risks inherent to any surgery, such problems include:

- Esthetic defect induced by forward movement of the maxilla

\section{INDICATIONS}

Molar distalization may thus be indicated in the following cases:

- To correct mandibular incisor alveolar protrusion, with or without associated crowding;

- To straighten a curve of Spee at the expense of the posterior sectors;

- Preoperative orthodontic preparation of class III compensation;

- Dental class III associated with skeletal class I malocclusion;

- Moderate skeletal class III, to be managed non-operatively by dentoalveolar compensation;

\section{UPDATE ON THE LITERATURE}

The Table below presents reports of results for molar distalization by pure lateral translation. with respect to the nose in Asian and African subjects;

- Postoperative discomfort that may be incompatible with the patient's private or occupational lifestyle (e.g., high level athlete);

- Psychological issues in patients unwilling to experience facial change, or fearing surgery;

- Financial cost of orthognathic surgery: $€ 2,000$ in France, $€ 50,000$ in Japan and $€ 80,000$ in the USA;

- Respiratory impact of mandibular recession and associated risk of obstructive sleep apnea syndrome.

An interesting possibility would be to correct class-III malocclusion by distalizing the mandibular molar while controlling the vertical dimension.

- Esthetic contraindications for class III correction by maxillary protraction in certain ethnic groups;

- Relative or absolute contraindications for orthognathic surgery;

- Mandibular alveolar asymmetry.

Finally, it should be borne in mind that posterior displacement of the mandibular molar cannot exceed the anatomic envelope within which it is possible: i.e., the mandibular lingual cortical bone.

According to Ridouani ${ }^{7}$ (Fig. 1), $3 \mathrm{~mm}$ distalization is the anatomic limit.

Briefly, no studies with high level of evidence and sufficiently reproducible design and results have 


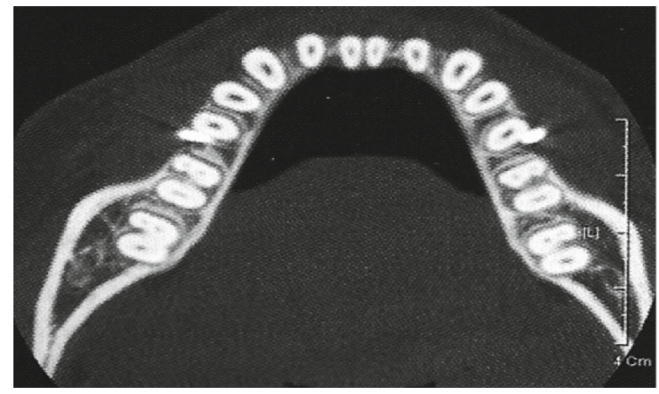

Figure 1

CT slice through the mandibular arcade after mandibular molar distalization by miniscrews ${ }^{7}$. Note contact between the distal 37 and 47 root and the lingual cortical bone of the mandibular body.

\begin{tabular}{|c|c|c|c|}
\hline Byloff et al. ${ }^{1} 2000$ & $\begin{array}{l}\text { Franzulum appliance } \\
\text { (Fig. } 2 a, b, c)\end{array}$ & Case report & $\begin{array}{l}\text { Non-significant single case; } \\
\text { Vestibular version effect on } \\
\text { mandibular incisors. }\end{array}$ \\
\hline Sugawara et al. ${ }^{8} 2004$ & $\begin{array}{l}\text { Distal osteosynthesis plate on } 7 \text {, chain } \\
\text { on } 4 \text { with plate or ligature of } 4 \text { with } \\
\text { plate and open spring in compression } \\
\text { (Fig. } 3 a \text {, b) }\end{array}$ & 15 case series & $\begin{array}{l}\text { Small series, } 2 \text { different protocols, } \\
\text { little detail of protocols. }\end{array}$ \\
\hline Hisano et al. ${ }^{4} 2007$ & TIM III (Fig. 4) & Case report & Non-significant single case. \\
\hline Lim et al. ${ }^{5} 2011$ & $\begin{array}{l}\text { Mini-screw between } 6 \text { and } 7 \\
\text { and sliding jig + chain (Fig. } 5 a, b, c)\end{array}$ & Case report & $\begin{array}{l}\text { Non-significant single case; } \\
\text { non-reproducible technique. }\end{array}$ \\
\hline
\end{tabular}

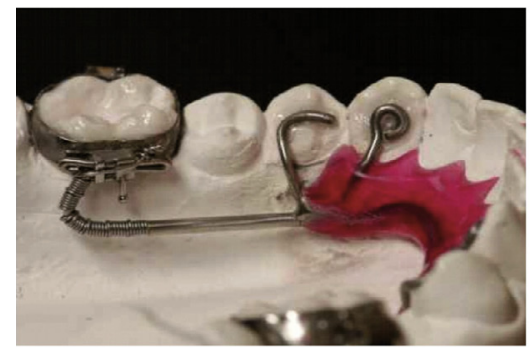

a

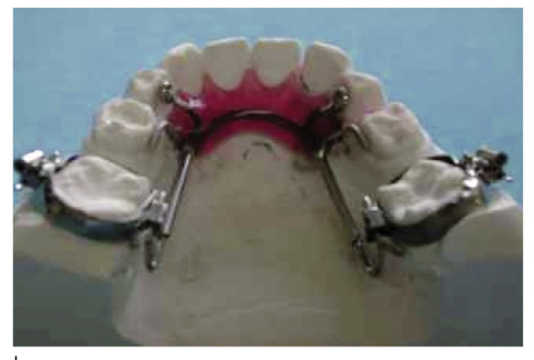

$\mathrm{b}$

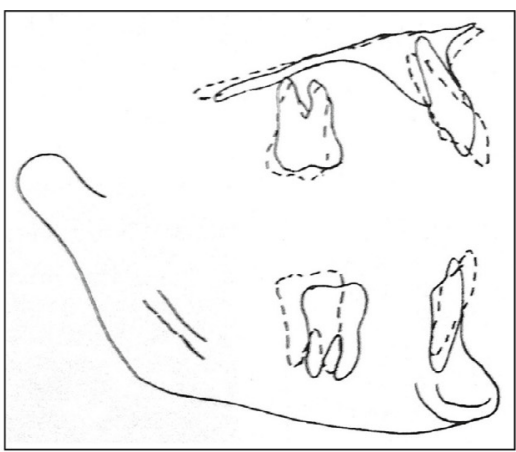

C

Figure 2

(a, b) Lingual view of Franzulum appliance on plaster model. (c) Cephalometric superimpositions obtained by Byloff et al. ${ }^{1}$ after molar distalization by Franzulum appliance. (See reproduction permissions at end of article.) 

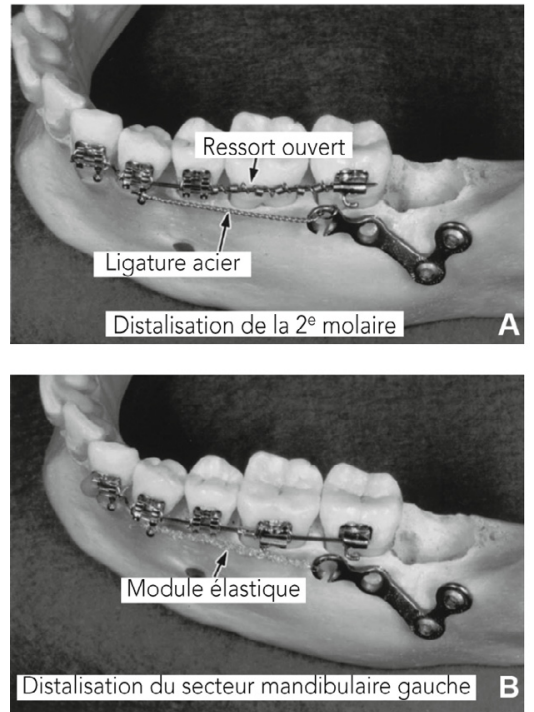

a

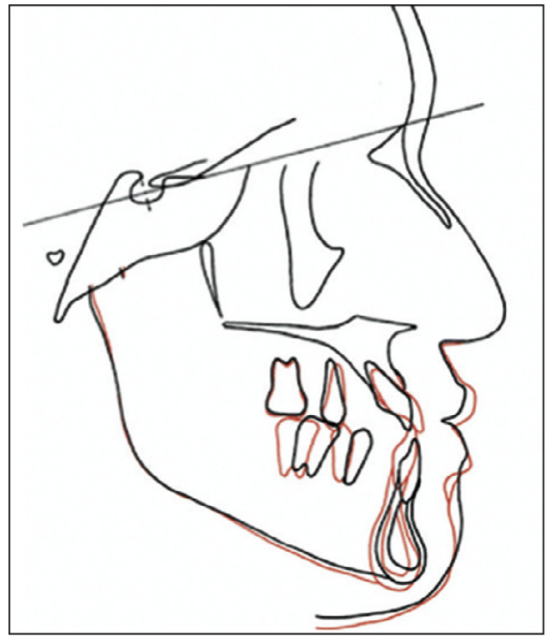

Phase 1

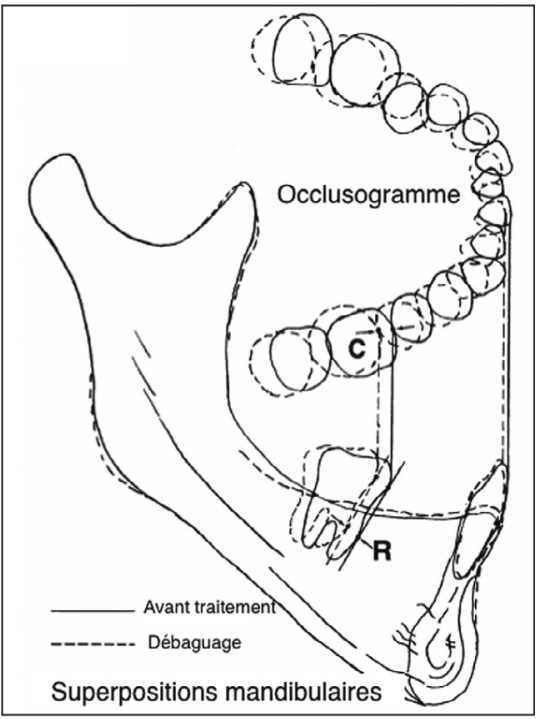

b
Figure 3

Sugawara et al.'s molar distalization protocol' ${ }^{8}$. A: unitary molar distalization; $B$ : sector distalization. (b) Cephalometric and occlusographic superimpositions of Sugawara et al.'s results ${ }^{8}$ (See reproduction permissions at end of article.)
Figure 4

Cephalometric superimpositions obtained by Hisano et al. ${ }^{4}$ : Phase 1 in black, phase 2 in red. (See reproduction permissions at end of article.)
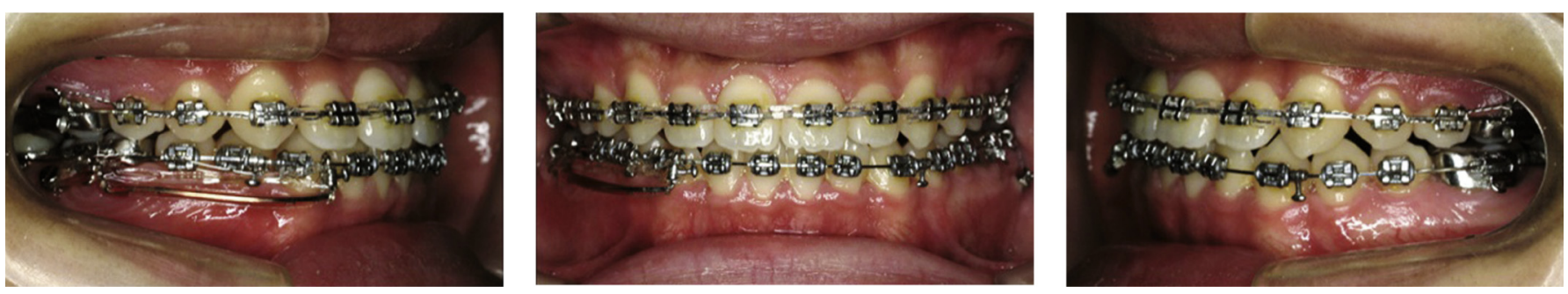

Figure $5 a$

Intra-oral photographs of Tai et al. 's ${ }^{9}$ molar distalization "sliding jig". (See reproduction permissions at end of article.) 


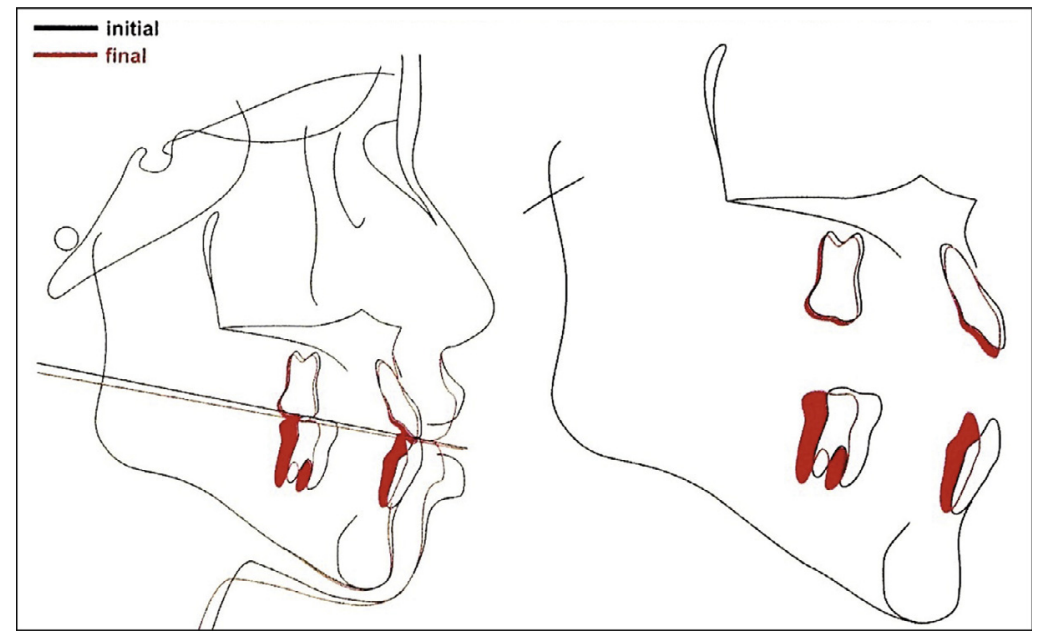

Figure 5b

Cephalometric superimpositions obtained by Lim et al. ${ }^{9}$ (See reproduction permissions at end of article.)

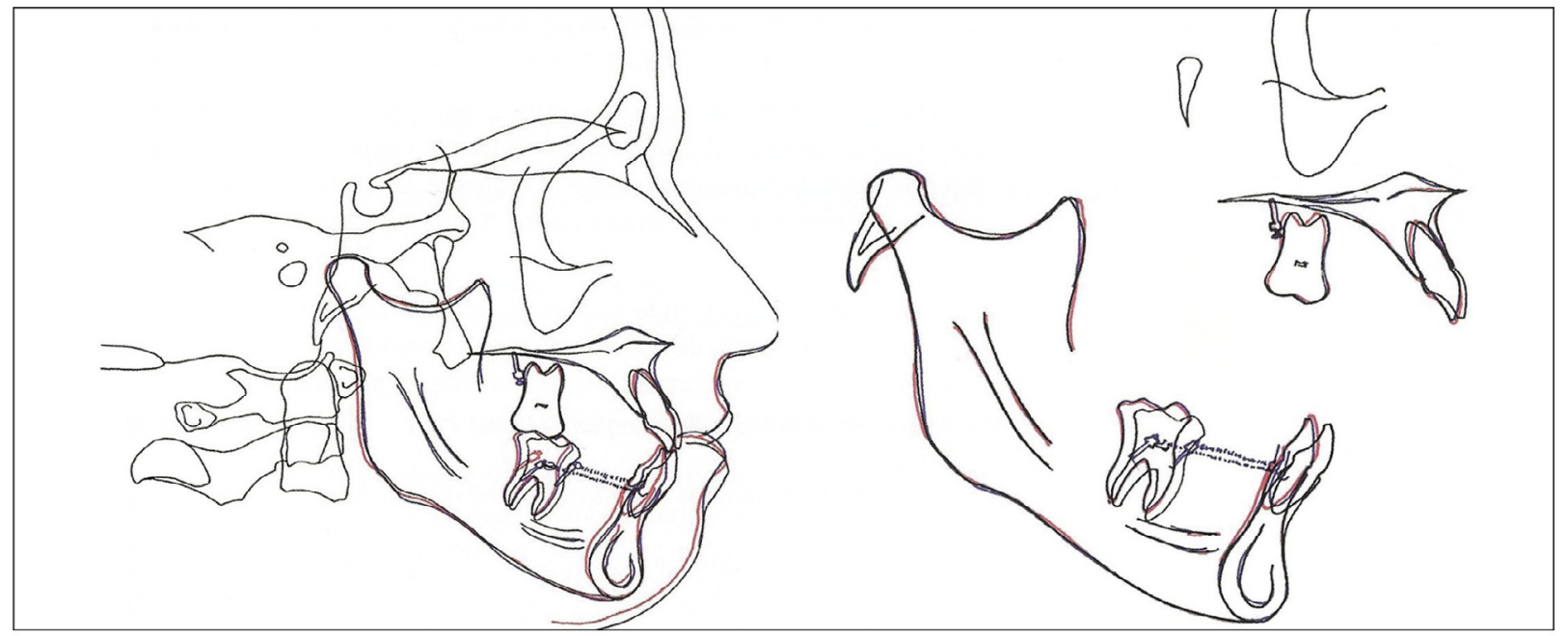

a
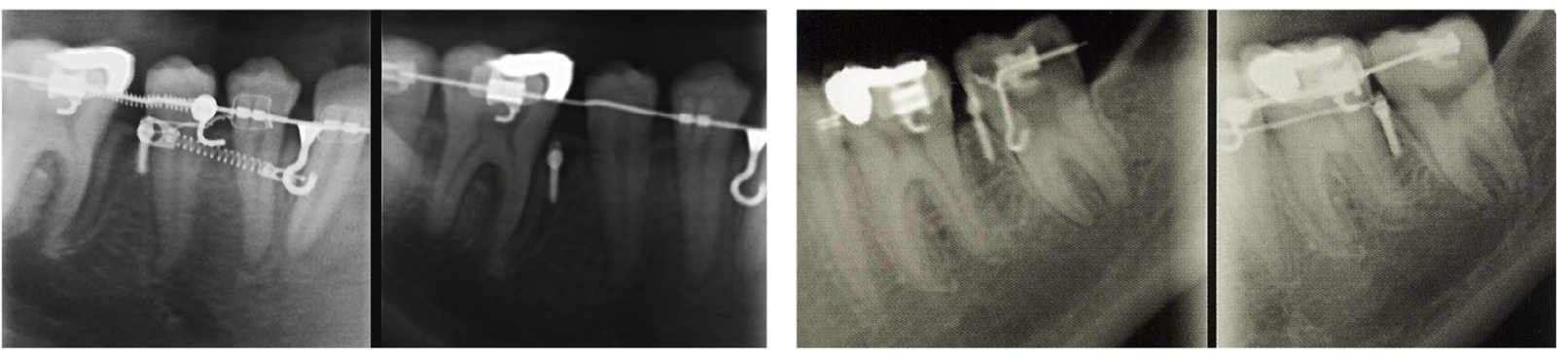

b

Figure 6

(a) General and local structural superimpositions by Ellouze and Darqué2 (Reproduced with editor's permission). Mandibular arcade distalization. Skeletal vertical control of hyperdivergence by vertical control of maxillary and mandibular molars. Slight compensatory protrusion of mandibular incisors. (b) Panoramic radiograph, by Ellouze and Darqué ${ }^{2}$ (Reproduced with editor's permission). 1: 46 distalization, showing trace of the initial position of the mesial root of the distalized molar. Displacement of mini-implant mesially to the distalized 46. 2: Surgical guide used to position mini-implant $(1.3 \times 7 \mathrm{~mm})$ between 36 and 37 and distalization of molars. 
identified a technique for mandibular molar distalization by pure lateral translation.

\section{CASE SERIES AND SUPERIMPOSITIONS}

\section{Material and methods}

Eleven cases of mandibular molar distalization were retrospectively analyzed, coming from three practitioners in private practice or hospital:

- 7 cases managed by Dr JeanPaul Forestier (JPF), in private practice in Paris;

- 2 cases managed by Dr Benoît Thebault (BT), in private practice in Redon (France);

- 2 cases managed by Dr Tho Dang (TD) under the supervision of Dr Jean-Paul Forestier in the Pitié-Salpêtrière Hospital, Paris.

Indications were:

- Correction of anterior mandibular crowding or of recurrence of anterior crowding;

- Decompensation of class II malocclusion for mandibular protraction surgery;

- Correction of dental class III;

- Straightening of 2 nd molars for alteration of prosthesis fixed to the 1st molar.

Exclusion criteria were:

- Simple coronal tip-back;

- Lack of post-distalization radiographs;

- Asymmetric distalization in which the distalized sector could not be identified on lateral teleradiograph for superimposition.
The most interesting study, by Sugawara ${ }^{8}$, included only 15 patients (30 distalizations), with 2 different protocols.

Cephalometric tracing of teleradiographs on the Delaire Evolution software involved tracing the orthognathic, topographic and dental Delaire analysis points. The software's "Compare" tool provides mandibular superimposition on the "No-Me" (notch and chin) axis, registered on the "Me" (chin) point, displaying the change in molar axis with respect to these references. The molar is shown by a cross, the mesiodistal axis of which is determined by the points "mim" (mesial inferior molar) and "mid" (distal inferior molar), and the long axis by the points "mio" (occlusal inferior molar) and "mia" (apical inferior molar).

The radiographs from the different radiology systems did not always include a millimeter scale; scaling was therefore harmonized using two points that were easily located and sufficiently separate: " $\mathrm{M}$ " (metanasion) and "Clp" (posterior clinoid process). Thus, measurements in millimeters could not be taken: displacement quality could be assessed but not quantified. Moreover, angles could not be measured as the software's angle measurement tool is not part of the "Compare" tool, so that angles cannot be measured in superimpositions.

Superimpositions were made on lateral teleradiographs taken before and after distalization. In some 

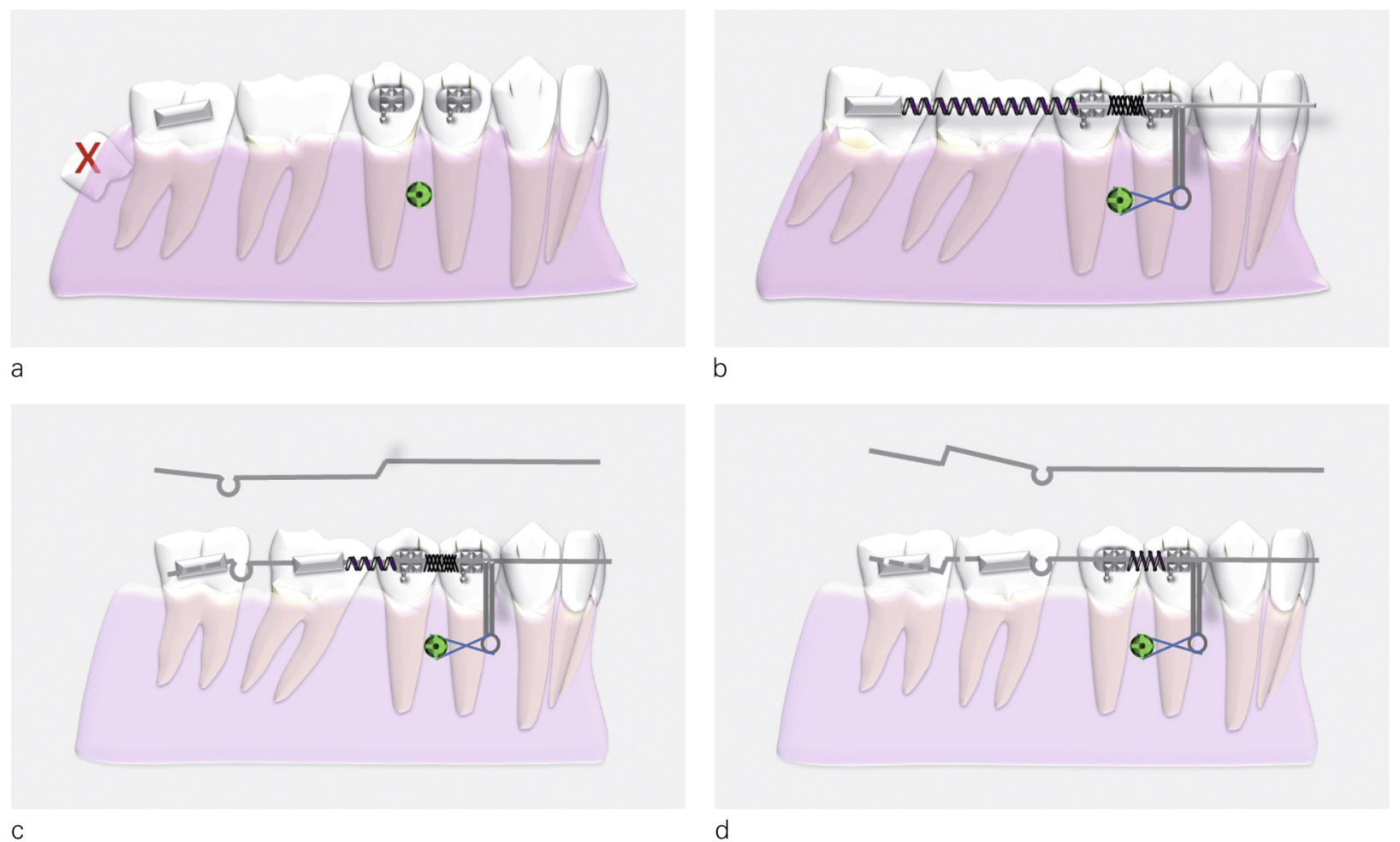

Figure $7 a, b, c, d$

cases, a third view was available, allowing supplementary superimposition.

\section{Protocols and clinical cases}

- Dr Jean-Paul Forestier's protocol for molar distalization with miniscrew anchorage (Fig. 7a, b, c, d).

- Gluing the mandibular arcade, with the exception of the incisors and canines; bracketing 1st molars with a pre-adjusted multiattachment .018 Hilgers bracket.

- Imposing tip-forward in molar gluing and bracketing.

- Straightening using $.016 \times .016$ NeoSentalloy ${ }^{\circledR}$ wires, bypassing incisors and canines.
- Extraction of mandibular wisdom teeth and positioning anchor screws between 4 and 5 (Fig. 7a).

- .016 x .022 Elgiloy ${ }^{\circledR}$ Jaune wire with open spring between 6 and 7, closed spring between 5 and 4 and mesial keyhole in 4 with metal ligature of the mini-screw to the keyhole. The mini-screw serves as indirect anchorage. Distalization is performed quickly after 38-48 extraction to take advantage of distal 37-47 bone remodeling and facilitate distal movement (Fig. 7b).

- Imposing tip-forward on the 6 glued teeth, and straightening by an underlay wire if necessary.

- The open spring is displaced progressively and the distalized teeth 


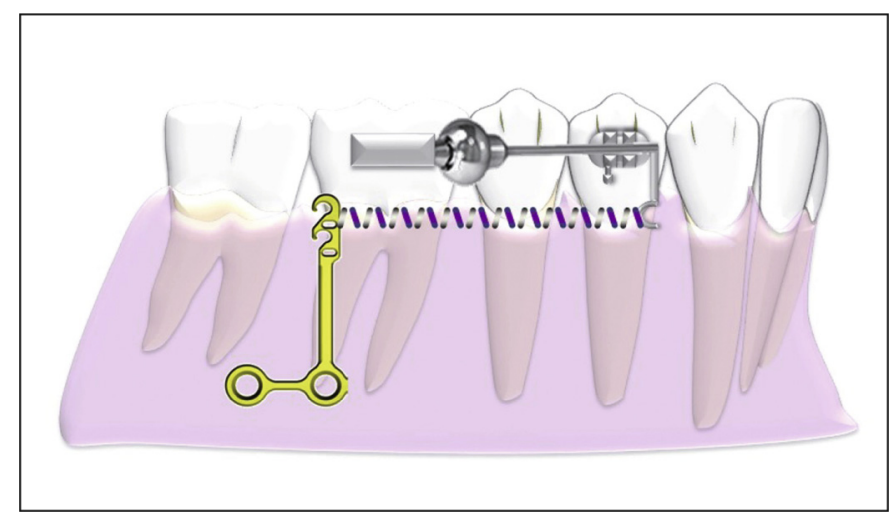

Figure $8 a$

Dr Thebault's Tekka ${ }^{\circledR}$ plate sectorial molar distalization with Distaler ${ }^{\circledR}$.

are blocked by an omega loop in contact with the distalized tooth. The intrusion step between 5 and 6 anticipates the protrusion of the mesial marginal crest of 6. Tipforward onto the wire helps distal repositioning of the apex of the tipped-back teeth (Fig. 7, c and d).

- Treatment of incisors and canines, when the space created by molar and premolar distalization permits, by a $.016 \times .016$ NeoSentalloy ${ }^{\circledR} \mathrm{NiTi}$ shape-memory wire and metal ligature between 4 and mini-screw.

- Progression toward a $.016 \times .022$ NeoSentalloy ${ }^{\circledR}$ then $.016 \times .022$ Elgiloy ${ }^{\circledR}$ Jaune wire.

If the screw was positioned distally to 37-47, a steel ligature using a .014 round wire at the screw neck emerges into the oral cavity along the vestibule, enabling, first, 34 and 44 to be blocked by an open spring when 7 is distalized, then the $7 \mathrm{~s}$ to be blocked when they are distalized, to fix their position.

\section{Dr Benoît Thebault's protocol for molar distalization with mini-screw anchorage}

- Patient 3 (Fig. 8 a, b)

- Extraction of mandibular wisdom teeth and positioning of Tekka ${ }^{\circledR}$ bone plate against the $6 \mathrm{~s}$.

- Gluing 4 and 6 with pre-adjusted Roth .018 slot multi-attachment bracket and positioning of Dista$\operatorname{ler}^{\circledR}$ (in the 6 lip-bumper sheath) with traction spring or chain between scaffold and direct anchorage plate (Fig. 8a).

- Treatment of 7s.

- Withdrawal of Distaler ${ }^{\circledR}$ once 6 and 7 distalization is sufficient.

- Progressive treatment of premolars then incisors and straightening by round NiTi wire and chain from plate to canine to maintain an anterior sector distalizing component during straightening.

- Progression up to $.017 \times .025$ steel wire (Fig. 8b). 

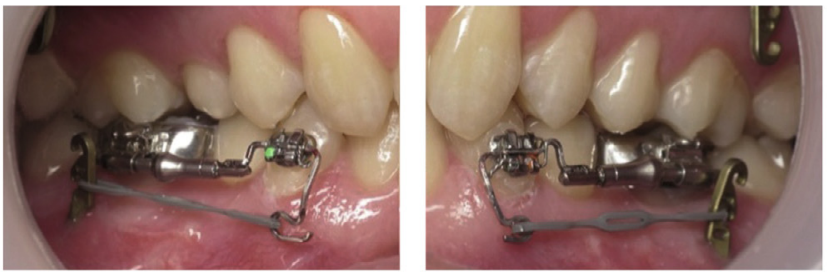

Figure $8 b$ Intra-oral photographs of one of Dr Thebault's patients. Sectorial molar distalization with Tekka ${ }^{\circledR}$ plate and Distaler ${ }^{\circledR}$.
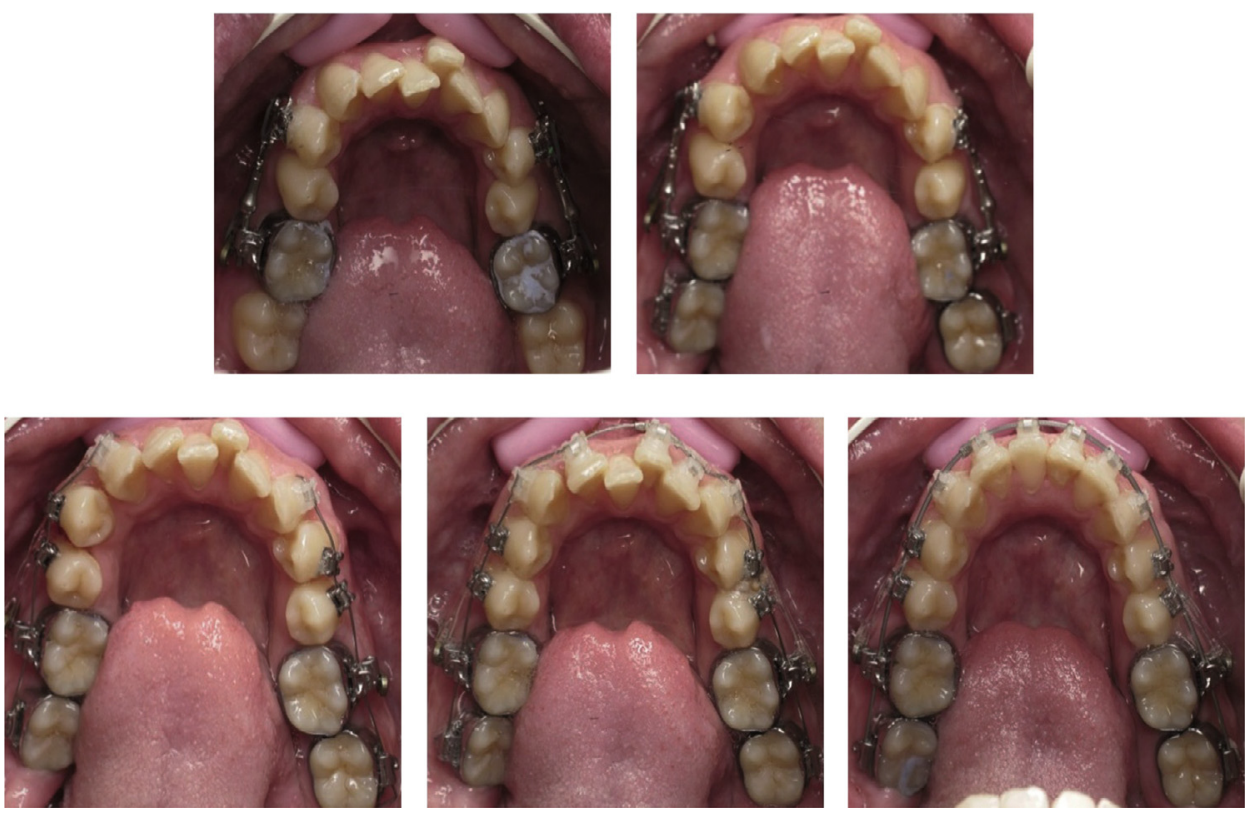
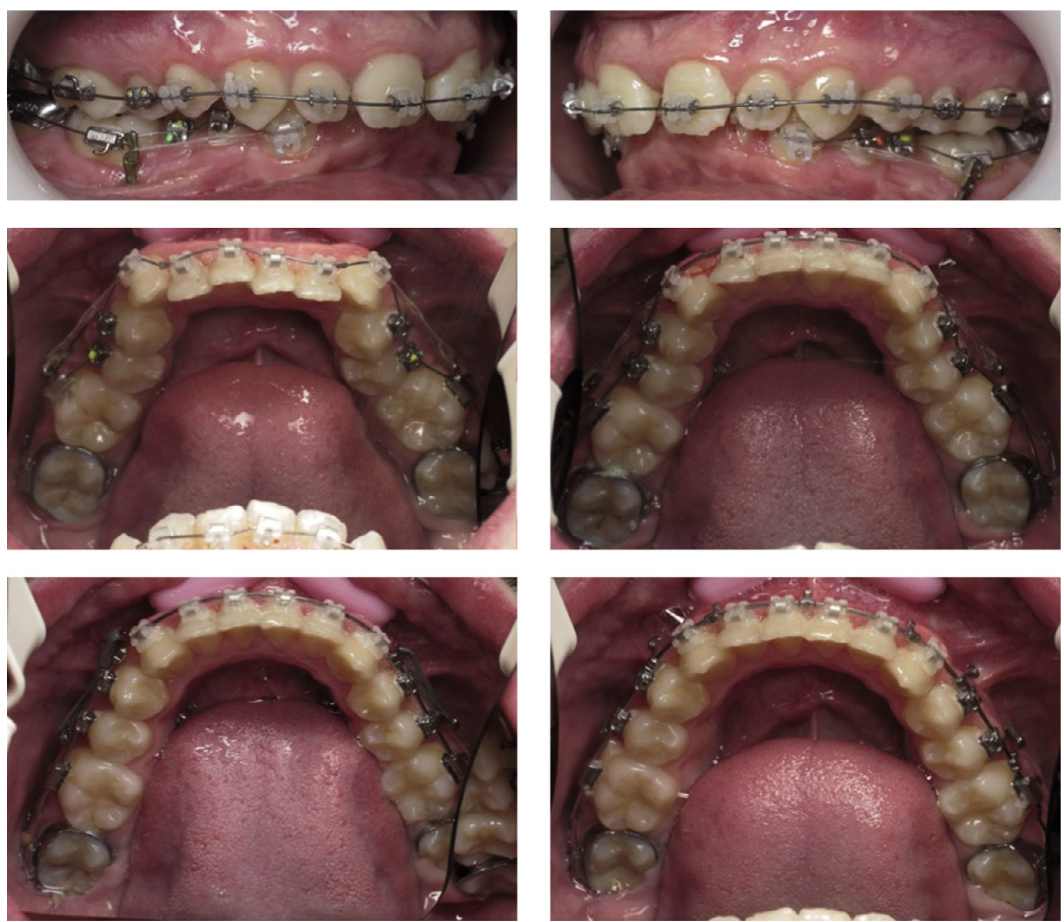

Figure 9

Intra-oral photographs of one of Dr Thebault's patients. Sectorial molar distalization with Tekka ${ }^{\circledR}$ plate. Note 37 and 47 crowns sunk into distal mucosa. 
- Patient 6 (Fig. 9)

- Gluing mandibular arcade and straightening with round $\mathrm{NiTi}$ wire up to $.017 \times .025 \mathrm{NiTi}$ wire, with chain between 3 and plate to maintain a distalizing component during straightening.

\section{RESULTS}

Table I on the next page present the results.

Summary of results: Table II.

Delaire cephalometric superimposition color code:

- Initial situation: red;

\section{DISCUSSION}

\section{Radiograph availability}

In some cases, radiographs were available for start of treatment and before avulsion (control).

In class II surgical cases, there were more radiographs due to the need for pre- and post-operative X-ray (up to 2 extra views).

\section{Lateral view timing}

In the case of patient 7, a lateral view taken 4 months after distalization of the crown showed apical repositioning, leaving time for the tipforward to manifest. In some cases, the end-of-treatment or postoperative radiograph showed no such apical repositioning, but in others, such as patient 9, there was no other view available showing whether coronary
- .0174 x .025 steel wire with long scaffold clipped on a wire between 3 and 4 and fitting a traction spring between scaffold and plate to act as direct anchor until the desired incisor repositioning is achieved.

- Situation after molar distalization: blue;

- When 3rd (postoperative) lateral teleradiograph available, final situation: green (Fig. 10).

distalization was accompanied by apical distalization.

Ideally, the protocol should prospectively define the best time-points for documenting the molar distalization, respecting the ALARA (As Low As Reasonably Achievable) principle, with the following sequence:

- Baseline documentation at start of treatment;

- Fitting the multi-attachment device, extracting wisdom teeth, positioning anchorage, with or without corticotomy;

- Start of molar distalization;

- End of molar distalization;

- Start of mesial tooth distalization;

- Then end-of-distalization record, late enough to allow apical repositioning; this interval is to be determined. 


\begin{tabular}{|c|c|c|c|c|c|c|}
\hline Patient & $\begin{array}{l}\text { Age at start } \\
\text { of treatment }\end{array}$ & Practitioner & Malocclusion & $\begin{array}{l}\text { Indication for molar } \\
\text { distalization }\end{array}$ & Anchorage & Result \\
\hline 1 & 16 years & JPF & $\begin{array}{l}\text { Class II division } 1 \\
\text { subdivision } \mathrm{G} \text {, and } \\
\text { mandibular } \\
\text { alveolar protrusion }\end{array}$ & $\begin{array}{l}\text { Sector } 4 \text { distalization, } \\
\text { for fitting } 43 \text { restraint } \\
\text { with conserved } 83\end{array}$ & $\begin{array}{l}\text { Extraction of } 48 \text { Mini- } \\
\text { screw between } 45 \\
\text { and } 46\end{array}$ & Apical tip-forward \\
\hline 2 & 42 years & JPF & Class II division 2 & Anterior crowding & $\begin{array}{l}\text { Extraction } 38-48 \\
\text { Mini-screw between } \\
4 \text { and } 5\end{array}$ & $\begin{array}{l}\text { Distalization by lateral } \\
\text { translation }\end{array}$ \\
\hline 3 & 25 years & BT & Class II division 2 & Anterior crowding & $\begin{array}{l}\text { Extraction } 34-48 \\
\text { Bone plate on } 6 \mathrm{~s}\end{array}$ & $\begin{array}{l}\text { Coronary and apical } \\
\text { distalization (coronary > } \\
\text { apical) + protrusion }\end{array}$ \\
\hline 4 & 17 years & JPF & Class II division 2 & $\begin{array}{l}\text { Anterior crowding and } \\
\text { decompensation before } \\
\text { surgery }\end{array}$ & $\begin{array}{l}\text { Extraction } 34-48 \\
\text { Bone plate on } 6 \mathrm{~s}\end{array}$ & $\begin{array}{l}\text { Coronary and apical } \\
\text { distalization (coronary > } \\
\text { apical) + intrusion }\end{array}$ \\
\hline 5 & 12 years & JPF & Class II division 2 & $\begin{array}{l}\text { Straightening curve of Spee } \\
\text { by posterior sector }\end{array}$ & $\begin{array}{l}\text { Extraction } 38-48 \\
\text { Mini-screws between } \\
44-45 \text { and } 35-36\end{array}$ & Coronary tip-back \\
\hline 6 & 18 years & BT & Class II division 2 & $\begin{array}{l}\text { Anterior crowding and } \\
\text { decompensation before } \\
\text { surgery }\end{array}$ & $\begin{array}{l}\text { Extraction } 38-48 \\
\text { Bone plate on } 6 \mathrm{~s}\end{array}$ & $\begin{array}{l}\text { Apical tip-forward } \\
\text { and intrusion }\end{array}$ \\
\hline 7 & 14 years & TD & Class I DMD & $\begin{array}{l}\text { Anterior crowding and } \\
\text { correction of class III }\end{array}$ & $\begin{array}{l}\text { Extraction } 38-48 \text { TIM III } \\
\text { on maxillary wire } \\
\text { Elgiloy Jaune } .017 \times .022 \\
+ \text { transpalatine wire }\end{array}$ & $\begin{array}{l}\text { Distalization by } \\
\text { lateral translation }\end{array}$ \\
\hline 8 & 35 years & JPF & Class I DMD & $\begin{array}{l}\text { Correction of anterior } \\
\text { crowding }\end{array}$ & $\begin{array}{l}\text { Distal bone screws } \\
\text { at } 47 \text { and } 37\end{array}$ & $\begin{array}{l}\text { Coronary tip-back } \\
\text { and protrusion }\end{array}$ \\
\hline 9 & 17 years & TD & $\begin{array}{l}\text { Class III subdivision } \\
\text { D }\end{array}$ & $\begin{array}{l}\text { Correction of class III in } \\
\text { sector } 4 \text { and anterior } \\
\text { crowding }\end{array}$ & $\begin{array}{l}\text { Extraction } 48 \\
\text { Distal mini-screw at } 47\end{array}$ & Coronary tip-back \\
\hline 10 & 36 years & JPF & Class II division 1 & $\begin{array}{l}37-47 \text { distalization to increase } \\
\text { mesio-distal diameter of } 36-46 \\
\text { implant-borne crowns, } \\
\text { decompensation before } \\
\text { mandibular protraction surgery }\end{array}$ & $\begin{array}{l}\text { Attachment glued } \\
\text { to } 36-46 \text { implant-borne } \\
\text { crowns }\end{array}$ & $\begin{array}{l}\text { Coronary and apical } \\
\text { tip-back (coronary > } \\
\text { apical) }\end{array}$ \\
\hline 11 & 30 years & JPF & $\begin{array}{l}\text { Class II division } \\
2 \text { DMD, } 35 \text { agenesis }\end{array}$ & $\begin{array}{l}\text { Anterior crowding and } \\
\text { incisor repositioning } \\
\text { before mandibular } \\
\text { protraction surgery }\end{array}$ & $\begin{array}{l}\text { Extraction of } 38-48-75 \\
\text { Distal mini-screw at } 47 \\
\text { and at agenesic } 35\end{array}$ & Coronary tip-back \\
\hline
\end{tabular}




\begin{tabular}{|l|c|}
\hline \multicolumn{1}{|c|}{ Movement achieved } & Number of patients \\
\hline $\begin{array}{l}\text { Distalization by lateral translation } \\
\text { (Fig. 10a) }\end{array}$ & 2 \\
\hline $\begin{array}{l}\text { Distalization + coronary tip-back } \\
\text { (Fig. 10b) }\end{array}$ & 3 \\
\hline Coronary tip-back (Fig. 10c) & 4 \\
\hline Coronary tip-forward (Fig. 10d) & 2 \\
\hline TOTAL & 11 \\
\hline
\end{tabular}

\section{Limitations of superimposition on lateral teleradiographs in Delaire analysis}

Asymmetric cases are necessarily excluded from 2D superimposition, as it is very difficult if not impossible to identify the molar to be tracked, especially when teeth are doubled or superimposed on another structure such as an anchor plate.

Table /I
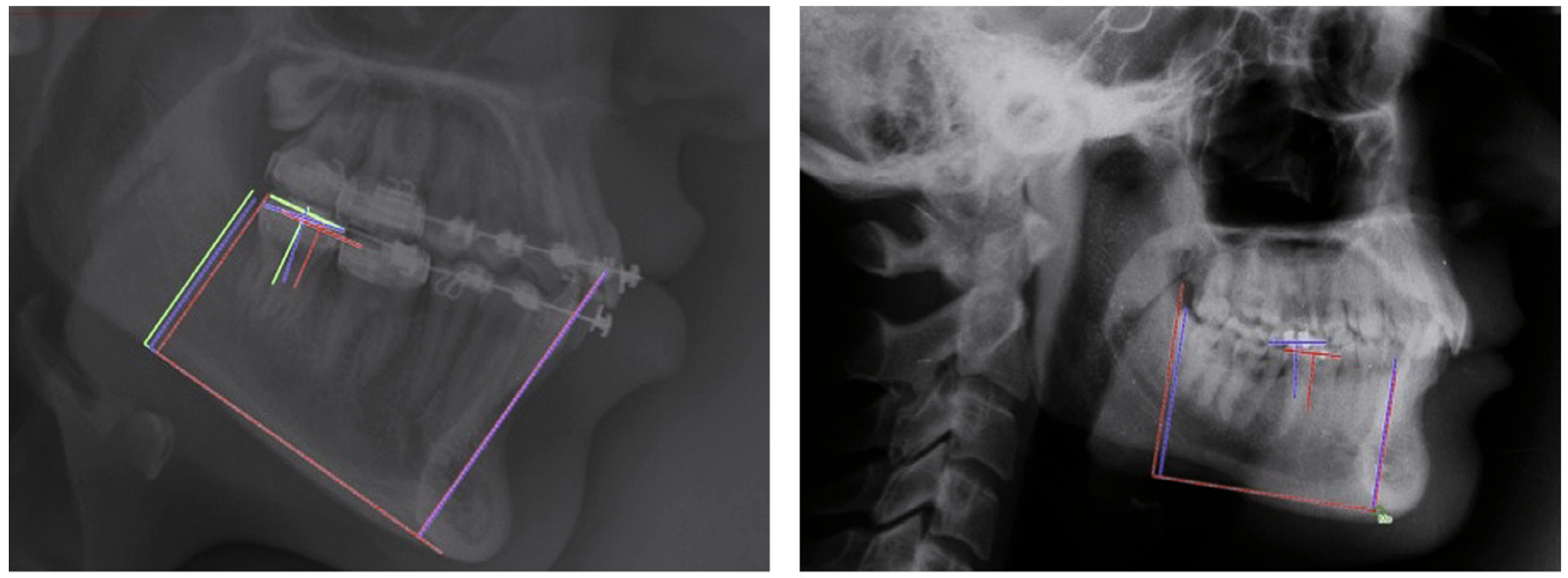

a

b
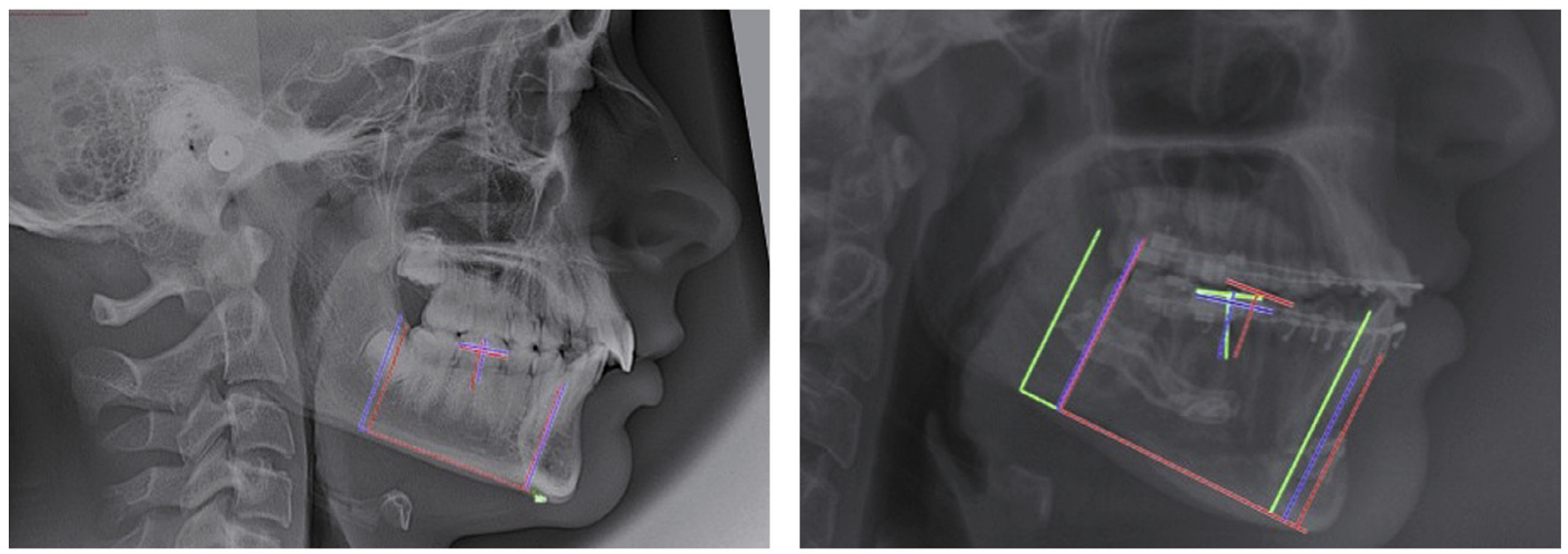

d

Figure 10

Delaire cephalometric superimpositions. (a) Distalization with pure lateral translation; (b) distalization and coronary tip-back; (c) apical tip-forward; (d) coronary tip-back only. 
Precise superimposition requires very high-quality X-ray, without doubling of anatomic structures other than in case of true asymmetry, using the same equipment, with sufficient quality to identify all anatomic structures. This was not always the case for the radiographs available. Some, moreover, had not been taken in a digital format, and a digital image had to be taken from $X$-rays displayed on a negatoscope; this entails parallax error and the kind of edge deformation encountered using a wide-angle lens. Some lateral teleradiographs were taken at $4 \mathrm{~m}$, others at $1.5 \mathrm{~m}$, making measurement impossible. The superimposition results were thus sometimes difficult to interpret. Scales could differ between treatment phases if the patient had changed radiologists or the radiologist had changed equipment. Cephalostat pitching and doubling of anatomic structures vitiated interpretation of vertical molar movement with respect to the basilar edge.

Moreover, 2D superimposition cannot reveal rotational movement during distalization, showing as reduced inter-radicular distance.

Furthermore, dental superimposition in Delaire analysis has the drawback of the teeth being represented by a cross corresponding to the occlusal side of the tooth and the long axis. The mandibular molar, however, may show very variable anatomy, with roots of varying length and apices that are more or less distal, so that there may be a certain vertical and mesiodistal margin of error in situating the "mia" point (apical inferior molar, between the roots of the $1 \mathrm{st}$ mandibular molar).

Comparison concerned in some cases the 1st molar, but in others the 2nd:

- Either due to absence of 36-46;

- Or because movement could be measured only on the 7s, which are easier to track than the 6 if an anchor plate is superimposed;

- Or because the lateral teleradiograph was taken after isolated distalization of 7 .

Furthermore, while superimposition on the "Me" (chin) point is unproblematic in mandibular protraction surgery, it comes up against its limitations if the patient has undergone genioplasty or changed position.

3D imaging with an orthonormal landmark based on fixed anatomic elements (Treil analysis) could get around these difficulties in assessing dental movement quality. But using such radiation for such a purpose is ethically dubious.

\section{Diversity of protocols and indications}

The one common point in the selected cases was that molar distalization was included in the treatment plan. Malocclusion, facial type (hypoor normo-divergent) and the objective of distalization, on the other hand, varied. Some cases showed tip-back only, or distalization by lateral translation associated with a tip-back component. The objective, however, 
could in some cases be to straighten the curve of Spee by coronary tipback, without necessarily seeking apical distalization.

Given the diversity in baseline malocclusion, dental formula, mini-screw sites and degree of crowding, the protocols implemented likewise differed, and successful distalization could not be attributed to one particular protocol. In 2 cases (patients 2 and 7), pure lateral translation was achieved, with very different protocols and anchorages. In other cases, results differed for the same protocol: apical tip-forward in patient 1, coronary tip-back in patient 4 after replacement of an infected left bone plate by a screw between 35 and 36. Finally, the objectives of molar positioning in class III correction do not require the same degree of distalization as would be needed for arcade preparation for mandibular protraction.

It is therefore difficult to draw conclusions from these findings, based on too small a sample with too diverse protocols, and to determine whether one device is more effective than another. The literature review encountered the same problem: apart from Sugawara's study (15 patients, 30 displacements), all the other publications were of case reports, with too low a level of evidence for any conclusions to be drawn as to the reliability of one system or another. Likewise, a given system used by two operators in two different patients might not give the same result (e.g., Tai et $a l^{9}$ vs Lim et $a l^{5}{ }^{5}$ ). However, the literature review concerned mainly class III correction in Asian populations, where it is more frequent than in Europe, where class $\|$ is more common. Moreover, in most cases what was presented was a case report.

\section{latrogenic effects of such dental movement, and long-term stability}

At end of treatment, certain cases showed radicular resorption in molars visible on panoramic X-ray. Although without clinical impact for the patient, this was visible on X-ray, and relatively unpredictable. Retrospectively, it was found to correspond to cases with no coronary movement but with apical tip-forward (patients 1 and 6). In case of movement in pure lateral translation, patient 2 (38 years of age) showed radicular resorption while patient 7 (14 years of age) did not. Age may perhaps be a factor for resorption.

Patient 8 displayed frontal shift of the occlusion plane during treatment, probably due to difference in height of the mini-screw anchorages. This possible side-effect had been overlooked.

Finally, the question of the longterm stability of these movements remains unresolved. None of the present cases allowed stability to be demonstrated, due to lack of data or of post-treatment follow-up; the last radiograph was, at best, a lateral teleradiograph taken after ablation.

\section{Protocols and implementation in dental chair}

In assessing the efficacy of the various protocols described, efficiency 
was not taken into account: some protocols are easier than others to implement in the clinical situation. Such factors include:

- Ease of implementation in dental chair: operator-dependent, or delegatable?

- Time-consumingness: self-ligaturing? Preformed arch?

- Number of, interval between and length of consultations;

- Materials costs, for practitioner and for patient;

- Patient cooperation requirements.

In practice, bone anchorage creates a submucosal entry portal, with consequent risk of infectious complications. Mini-screws may become detached and have to be ablated and reinserted, necessarily in another site. Patient 8 complained of jugal discomfort throughout his treatment, due to screw protrusion. Screwrelated complications included adjacent cyst, occurring in about $6 \%$ of cases. Patient 4 experienced bone plate infection, requiring replacement of the plate by a screw. Bone plates are associated with a chronic inflammation rate exceeding $7 \%{ }^{10}$.

Ablating a screw is straightforward, but ablating an anchorage plate requires surgical revision and a further flap.

Moreover, including arcade distalization in a treatment plan requires longer treatment than class III orthosurgical correction or Triaca frontblock distraction ${ }^{6}$ to correct anterior crowding. Likewise, the comparative risk/benefit-ratio of extracting premolars adjacent to the crowded sector versus extracting wisdom teeth and distalizing the posterior teeth to correct anterior crowding needs to be assessed in terms of length of treatment and risk of complications.

It should be noted that temporary anchorages require good coordination between the practitioner extracting the wisdom teeth, who will also fit the anchors, and the orthodontist, who should quickly initiate molar distalization so as to take advantage of the bone remodeling induced by extraction.

Finally, one adverse effect of molar distalization concerns access to the wire distal to the 2nd molars, which regularly sink under the retromolar mucosa, preventing access to the distal side of the 7 tube, which may have to be shortened, causing discomfort for the patient at each manipulation, and sometimes preventing ablation unless the mucosal covering is lifted.

All in all, comparing anchorage by mini-screw or mini-plate versus classIII elastic anchorage shows that lateral translation can be achieved either way (patients 2 and 7). However, in the latter case the orthodontist requires the patient's cooperation and imposes on the temporomandibular joints, the elastic bands having to be worn constantly, while the former makes no requirements of cooperation but, in 10\% of cases, induces complications relating to the bone anchorages ${ }^{10}$. 


\section{CONCLUSION}

In 2014, mandibular molar distalization appeared feasible. Several issues, however, remain in suspense:

- Which protocol, providing optimal reproducibility, has proved effective in a sufficiently large sample?

- With what efficiency?

- With what iatrogenic effects? Can they be quantified, Can they be predicted?

- How stable are results over the long term, notably in skeletal class III correction?

A prospective comparative randomized study will be needed to test the efficacy of each protocol in specific clinical situations of malocclusion, of quality and quantity of planned movement, with similar facial type, and with a sufficiently large sample to assess results on 3D CT or conebeam measurement so as to overcome the drawbacks of 2D cephalometry. The number of acquisitions and the corresponding interval(s) will need to be determined in advance to allow study of dental movement during treatment. Comparison should include efficiency from the practitioner's point of view and iatrogenesis from the patient's.

\section{Reproduction permission}

Figure 2: Taken from J Clin Orthod, 34, Byloff F, Darendeliler MA, Stoff F,
Mandibular molar distaliza- tion with the Frangulum Appliance, 518-523; Figure 7, Copyright 2000.

Figure 3: Taken from Am J Orthod Dentofacial Orthop, 125, J Sugawara et al., Distal movement of mandibular molars, in adult patients with the skeletal anchorage system, 9 pages, Copyright 2004, with permission from Elsevier.

Figure 4: Taken from Am J Orthod Dentofacial Orthop, 131, Hisano M, Chung CJ, Soma K, Nonsurgical correction of skeletal Class III malocclusion with lateral shift in an adult, chapter 6, 8 pages, Copyright 2007, with permission from Elsevier.

Figure 5a: Taken from Am J Orthod Dentofacial Orthop, 144, Tai K, Park JM, Tatamiya M, Kojima Y, Distal movement of the mandibular dentition with temporary skeletal anchorage devices to correct a class III malocclusion, 10 pages, Copyright 2013, with permission from Elsevier.

Figure 5b: Taken from $\mathrm{J}$ Clin Orthod, 45, Lim J-K, Jeon MJ, Kim JH, Molar distalization with a miniscrewanchored sliding jig, 368-377; Figure 9b, Copyright 2011.

Conflicts of interest: The author declares no conflict of interest.

\section{BIBLIOGRAPHY}

1. Byloff F, Darendeliler MA, Stoff F. Mandibular molar distalization with the Franzulum Appliance. J Clin Orthod 2000;34(9):518-23. 
2. Ellouze S, Darque F. Gestion orthodontique des asymétries d'arcade. MINIIMPLANTS/ L'orthodontie de demain. Paris : Quintessence International 2012:147-82.

3. Furstman L, Bernick S, Aldrich D. Differential response incident to tooth movement. Am J Orthod 1971;59(6):600-8.

4. Hisano M, Chung CJ, Soma K. Nonsurgical correction of skeletal Class III malocclusion with lateral shift in an adult. Am J Orthod Dentofacial Orthop 2007;131(6):797-804.

5. Lim J-K, Jeon HJ, Kim JH. Molar distalization with a miniscrew-anchored sliding jig. $\mathrm{J}$ Clin Orthod 2011;45(7):368-77.

6. Merli M, Merli M, Triaca A, Esposito M. Segmental distraction osteogenesis of the anterior mandible for improving facial esthetics. Preliminary results. World J Orthod 2007;8(1):19-29.

7. Ridouani M. Mémoire de D.U.O. Insertion des miniimplants en parallèle des molaires mandibulaires: évaluation tomodensitométrique. Bordeaux 2-Victor Segalen 2013.

8. Sugawara J, et al. Distal movement of mandibular molars in adult patients with the skeletal anchorage system. Am J Orthod Dentofacial Orthop 2004;125(2):130-8.

9. Tai K, Park JH, Tatamiya M, Kojima Y. Distal movement of the mandibular dentition with temporary skeletal anchorage devices to correct a Class III malocclusion. Am J Orthod Dentofacial Orthop 2013;144(5):715-25.

10. Takaki $\mathrm{T}$, et al. Clinical study of temporary anchorage devices for orthodontic treatment-stability of micro/mini-screws and mini-plates: experience with 455 cases. Bull Tokyo Dent Coll 2010;51(3):151-63. 